\begin{tabular}{|c|c|c|}
\hline & $\begin{array}{l}\text { European Association for the Development of } \\
\text { Renewable Energies, Environment and Power Quality } \\
\text { (EA4EPQ) }\end{array}$ & $\begin{array}{l}\text { International Conference on Renewable Energies and Power Quality } \\
\text { (ICREPQ'12) } \\
\text { Santiago de Compostela (Spain), 28th to 30th March, } 2012\end{array}$ \\
\hline
\end{tabular}

\title{
Analysis and Characterization of a Square-Wave Modulation Method for Single- Phase Cascaded H-Bridge Multilevel Inverters
}

\author{
João C. Faria ${ }^{1}$ and António P. Martins ${ }^{2}$ \\ 1,2 Department of Electrical and Computer Engineering \\ Faculty of Engineering, University of Porto \\ Rua Dr Roberto Frias, s/n, 4200-465 Porto (Portugal) \\ Phone/Fax number: +351 225081816, e-mail: ajm@fe.up.pt
}

\begin{abstract}
Cascaded multilevel inverters offer several advantages over other multilevel structures: modularity, high voltage capability, reliability. Additionally, a large number of cascaded inverters using a square wave modulation method allow low losses in the inverter and low harmonic distortion in the output voltage. This paper analyzes the main features of one of these modulation methods especially in what concerns to realtime implementation in a FPGA, output voltage distortion in single- and three-phase systems and modulation range in static and dynamic conditions. Also, scalability is addressed in order to evaluate the method's capability to be used in high voltage applications. Simulation results with a different number of $\mathrm{H}-$ bridge inverters are presented and they allow obtaining the main conclusions; experimental results verify and support those conclusions.
\end{abstract}

\section{Key words}

Cascaded inverters, Harmonic distortion, Multilevel inverters, Square-wave modulation

\section{Introduction}

Unlike conventional DC/AC inverters multilevel inverters are characterized by synthesizing a voltage waveform constituted by several steps, closer to a sinewave and so with a lower harmonic content. In multilevel topologies, the alternating voltages are obtained through the connection of several semiconductors that block or conduct a portion of the input power (current or voltage) and create several steps in the output voltages.

This semiconductor connection also allows in multilevel topologies to surpass other disadvantages, besides the lower harmonic content of the output voltage, occurring in conventional topologies, e.g. reduction of losses for the same switching frequency with less commutations; usage of switches with lower power capability and, so, cheaper; reduction of the $\mathrm{dv} / \mathrm{dt}$ switching; reduction of common- mode voltages; and substantial reduction of electromagnetic interference (EMI) levels. These last properties reduce, or even eliminate the need of input and output filters what represents also an increased efficiency [1], [2], [3]. However, multilevel topologies also have disadvantages like an increased number of semiconductor switches, more passive elements or diodes, and more complex control algorithms. In both conventional and multilevel inverters the harmonic content reduces with an increasing switching frequency but it is important to refer that the switching frequency influences the semiconductor losses and is limited by their technology.

A large number of derivations of the basic multilevel structure principle have emerged due to the different possibilities of combining the capacitive DC sources in order to generate multilevel outputs, [1]. Despite that, three main topologies are usually considered in the literature from the practical point of view: diode clamped, flying capacitors, and cascaded H-bridge, all in singleand three-phase configurations.

This paper deals with the single-phase cascaded H-bridge topology. Due to its highly modular structure this topology is particularly suitable for medium and high power applications reaching very high voltages and synthesizing a waveform with a very low harmonic distortion [4], [5]. The very low total harmonic distortion (THD) is obtained using fundamental frequency switching and so keeps switching losses at the lowest levels [4], [6].

The next section discusses modulation methods for cascaded H-bridge inverters: PWM and fundamental frequency switching (FFS). It also reviews two FFS methods in the context of real-time implementation capability. Section 3 presents simulation results and characterizes the low-frequency harmonics and THD of the output voltage, while Section 4 shows experimental 
results for an eleven-level inverter and discusses the method's main features. Finally, Section 5 discusses the main conclusions of the work.

\section{Cascaded H-Bridge Inverters}

This topology uses, at least, two conventional full-bridge single-phase inverters, usually designated H-bridges, connected in series, as shown in Fig. 1. Without more phases the topology constitutes a single-phase inverter.



Fig. 1. Power structure of a cascaded H-bridge inverter.

The topology needs each H-bridge to be supplied by an isolated DC source. One bridge has three levels in the output voltage $(E, 0$ and $-E)$; thus, in order to obtain a higher number of levels and a waveform with better quality it is needed more bridges connected in series.

For equal DC sources, the number of levels, $N_{L}$, in the output voltage is given by (1), being $S$ the number of $\mathrm{H}$ bridges of the multilevel inverter.

$$
N_{L}=2 S+1
$$

A higher number of levels can be obtained using DC sources with different and specific voltage levels, [1]. For example, with $E_{1}=E$ and $E_{2}=3 E$, a 9-level waveform can be synthesized instead of a 5-level one, with $E_{1}=E_{2}=E$.

In terms of power structure the cascaded H-bridge inverter (with each bridge supplied by an active power source) has the following advantages:

- There is no need of input DC-capacitor voltage balancing

- No neutral-point connecting diodes are employed or floating capacitors

- High modularity because for and increasing in the number of levels it is only needed to add H-bridges.

Some disadvantages, however, should be referred:

- It needs independent DC sources for each bridge, thus limiting its use in some applications

- The connection of isolated sources in bidirectional $\mathrm{AC} / \mathrm{DC} / \mathrm{AC}$ systems is not possible, short circuits occur; it is needed synchronous switching to avoid them.

\section{A. Switching Frequency}

Because switching losses are one of the biggest parts of the losses in a converter and being them dependent on the switching frequency it makes this factor one the most important ones to be considered in the choice of a switching/modulation method.

PWM switching techniques for multilevel inverters are an extension of the same principle for conventional inverters but with several carriers; they are usually designated multicarrier PWM, [2]. For cascaded single-phase inverters the level-shift PWM and the phase-shift PWM (PSPWM) can be used but the PSPWM has a lower harmonic content, [2]. A better waveform quality and higher bandwidth makes PWM switching preferable for low-power and high dynamics applications. However, low-frequency switching algorithms are desirable for high-power applications where switching losses must be kept low.

\section{B. Square-Wave Modulation}

This technique can be easily applied to CHB inverters due to their unique structure [1]. The operating principle is depicted in Fig. 2 for a seven-level inverter (three bridges) where $\theta_{\mathrm{k}}$ is the switching angle of the inverter and $E$ is the DC voltage.

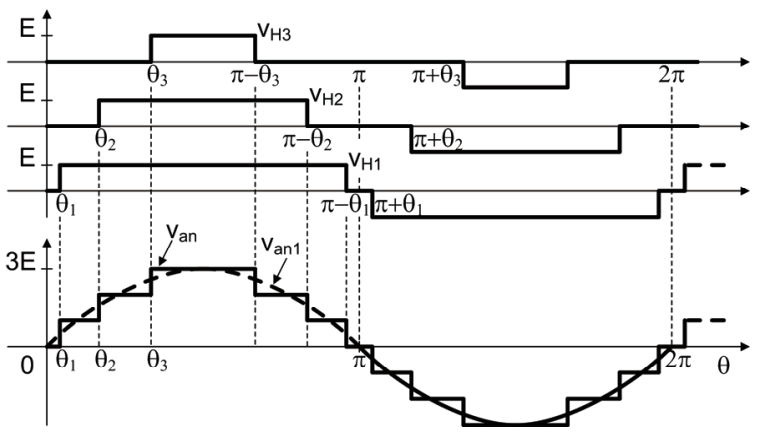

Fig. 2. Operating principle of the square-wave modulation.

Fundamental frequency switching, or square wave modulation, can be implemented pursuing different objectives, [7], [8], [9], [10], [12]:

- Minimization of the inverter output voltage THD, namely in photovoltaic applications

- Elimination of low-frequency harmonics of the output voltage, e.g. third, fifth, seventh ... harmonics, in applications where an efficient filtering of the remaining harmonics exist

- Minimization of the inverter output voltage harmonic content ignoring the third harmonic, cancelled in threephase systems

- Elimination of low-frequency harmonics ignoring the third harmonic.

In any case, the magnitude of the fundamental component must be controlled additionally to the optimization of the output voltage waveform and the switching angles are the means to do that. The output voltage, $v_{a n}$, of a cascaded Hbridge inverter with $S$ bridges can be expressed using the Fourier series expansion: 


$$
v_{a n}(t)=\frac{4 E}{\pi} \sum_{n=1}^{\infty}\left\{\frac{1}{n} \sum_{k=1}^{S}\left[\cos \left(n \theta_{k}\right)\right] \cdot \sin (n \omega t)\right\}
$$

subjected to $0<\theta_{1}<\theta_{2}<\ldots<\theta_{S}<\pi / 2$.

From the Fourier series, expressions relating the switching angles with a specific minimization objective are obtained. The amplitude modulation index is defined as

$$
m_{a}=\frac{V_{a n 1 \max }}{S \cdot 4 E / \pi}
$$

where $V_{\text {an } 1 \max }$ is the maximum value of the fundamental component and $S$ is the number of H-bridges. As an example, and using three bridges, if it is wanted to control the fundamental and eliminate the third and fifth harmonics the following equation system is obtained:

$$
\left\{\begin{aligned}
\cos \theta_{1}+\cos \theta_{2}+\cos \theta_{3} & =3 m_{a} \\
\cos 3 \theta_{1}+\cos 3 \theta_{2}+\cos 3 \theta_{3} & =0 \\
\cos 5 \theta_{1}+\cos 5 \theta_{2}+\cos 5 \theta_{3} & =0
\end{aligned}\right.
$$

From the point of view of computational complexity, algorithms for calculating the switching angles can be divided into two categories: non-real-time algorithms and real-time ones, [13].

In non-real-time algorithms the non-linear and transcendental equations are solved and the results saved in memory for later use in the control of the inverter. Different methods are possible such as: Newton-Raphson method, [5], method of resultant polynomials, [10], genetic algorithms, [14], particle swarm optimization, [15]. However, the computational effort and execution time do not allow these methods to be implemented in real-time in a microprocessor or a DSP. Also, for a higher number of levels the number of equations increases and in addition the required computational effort. Usually, these angles are calculated a priori for different modulation indexes by a computer with a high processing capability and stored in the chosen control platform by means of look-up tables. Moreover, if the DC sources of the various modules are different or variable the look-up tables must contain the different sets of solutions for all the combinations of input voltages and modulation indexes. The size of the tables is also strongly dependent on the wanted resolution of the modulation index.

In real-time algorithms several approaches can be found to avoid the resolution of the non-linear and transcendental equations: theory of balancing the voltage-time area of the reference voltage in relation to the output voltage, [11]; by analytical approximation of the THD expression, [12].

The method presented in [12] is chosen for demonstration in this paper. It implements the minimization of $\mathrm{THD}^{2}$ of the output voltage instead of THD. In [12], by simplification and equivalence, the expression of $\mathrm{THD}^{2}$ for a set of $S$ bridges is minimized and a closed expression for an auxiliary variable, $\rho$, from which all switching angles depend, is obtained. The real-time algorithm uses the two following steps:

1. Determine $\rho$ by solving (e.g. using the NewtonRaphson method) the equation:

$$
0=\sum_{k=1}^{S} \sqrt{1-\left(\frac{k-1 / 2}{S-1 / 2} \rho\right)^{2}}-m_{a} \cdot S
$$

2. Determine the switching angles by evaluating

$$
\theta_{k}=\arcsin \left(\frac{k-1 / 2}{S-1 / 2} \rho\right), \quad k=1,2, \ldots, S .
$$

In [13], this method is expanded for different or timevarying supplying voltages of each $\mathrm{H}$-bridge module. In all methods there is no control of the non-eliminated or minimized harmonics that usually tend to increase once the harmonic energy is redistributed by the non-eliminated harmonics.

The Newton algorithm is used to solve the non-linear equation because it assures fast convergence when a "good" initial solution is supplied, thus allowing the calculation of the switching angles in real-time. In addition to the initial solution several factors have influence in the speed of convergence of the method like the behaviour of function (5) and its derivative around the solution. The study of the graphic representation of $f(\rho)$ allowed establishing the limits where there are no solutions for the variation of the modulation index and also for the variation of the number of bridges of the inverter. For example, for $S=3, m_{a}$ must be higher than 0.6 ; for $S=7, m_{a}$ must be higher than 0.72 . In certain applications this is, of course, a strong limitation.

\section{Main Features of the Method}

In any inverter the output voltage must be controllable both in magnitude and frequency. Frequency control is usually simple depending only on the efficient use of timers. Magnitude control, both in range and dynamics, is more demanding. As pointed out in Section 2, the range of the fundamental component of the output voltage is a characteristic of the modulation method and the inverter power structure.

An inverter with a variable number of H-bridges was simulated and experimentally implemented. The first step was the validation of the real-time characteristic of the modulation method in the simulation environment. A script written in $\mathrm{C}$ was implemented in PSIM and the number of iterations has been counted during fast and small changes of the modulation index, maintaining a fundamental frequency of $50 \mathrm{~Hz}$. With $5 \mathrm{H}$-bridges the control algorithm was tested in two conditions: slow and fast variation of the modulation index, from $m_{a}=0.73$ to 0.98 in $40 \mathrm{~ms}$ and $2 \mathrm{~ms}$, and from $m_{a}=0.98$ to 0.73 in 2 $\mathrm{ms}$, as shown in Fig. 3.

As can be seen, the switching angles are updated almost instantaneously in both tests (in fact the Newton method takes four iterations to converge). The maximum number of iterations the method takes to converge for different conditions, and so the required absolute time, depends on specific hardware and will be discussed in the experimental implementation section. 




Fig. 3. Modulation index variation, switching angles and output voltage for slow and fast changes using $5 \mathrm{H}$-bridges.

Since the algorithm only minimizes THD and does not eliminate any specific harmonics one of its fundamental properties is the behaviour of the low-frequency harmonics, which will cause distortion in the output current. Figures 4 and 5 show the amplitude of all odd harmonics until the $19^{\text {th }}$, for the complete range of the modulation index: multiples of 3 , which will be cancelled in a three-phase system (Fig. 4) and non-triplen harmonics (Fig. 5).

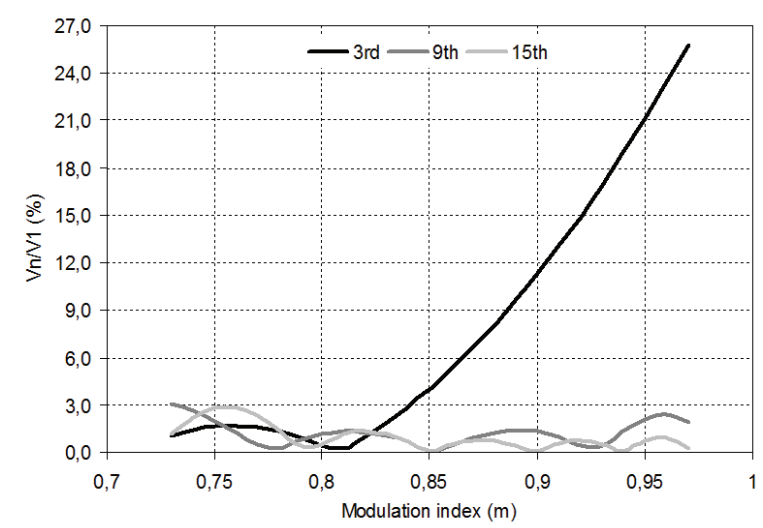

Fig. 4. Normalized amplitude of odd harmonics multiples of three for $5 \mathrm{H}$-bridges.

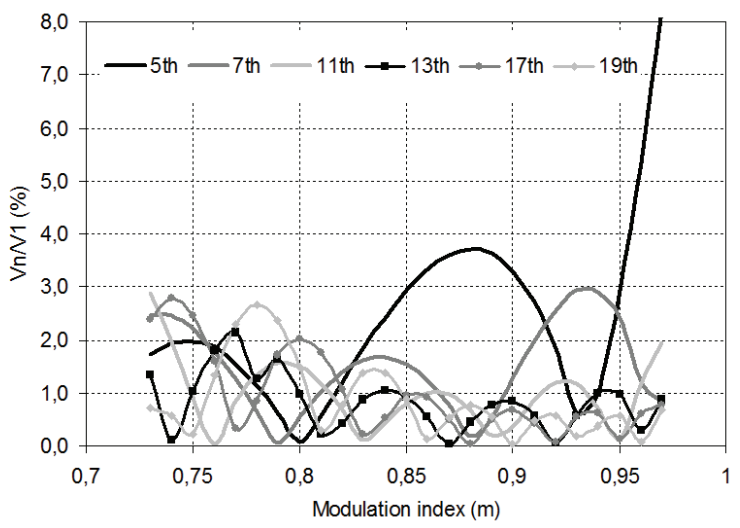

Fig. 5. Normalized amplitude of odd harmonics non-multiples of three for $5 \mathrm{H}$-bridges.
The THD of the output voltage is shown in Fig. 6 and reflects the behaviour of low order harmonics, mainly the third and the fifth.



Fig. 6. THD of the output voltage for 5 H-bridges.

For $m_{a}$ lower than certain levels the method does not provide a numerical solution and for high values of $m_{a}$ the THD gets high values, almost not usable in practical applications. So, it is considered a zone of "normal operation" of the method which is narrower for inverters with a larger number of levels (see Section 4.1 for additional discussion).

\section{Hardware Implementation}

The used hardware is represented in Fig. 7 and 8 (schematic diagram and photograph of the experimental set-up, respectively). A Xilinx Spartan 3-AN FGPA board, incorporating a MicroBlaze processor, was used to implement all the process of calculating the switching angles for the five inverters. In this section the presented results are centred in the characterization of the modulation method for an increasing number of levels. The parameters to be analyzed are the same already presented in the simulation section: evolution of the loworder harmonics with the modulation index (Fig. 9 and 10) and its limits for normal operation, i.e. the limits of the modulation index where an increasing in its value produces an increasing in the output voltage fundamental component.

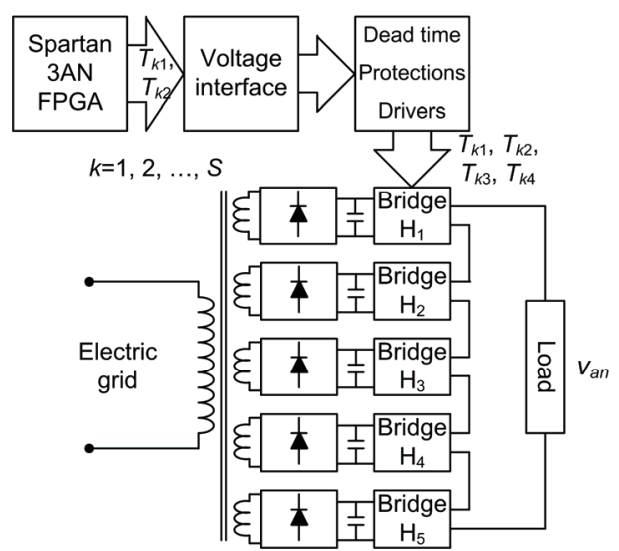

Fig. 7. Schematic diagram of the experimental set-up. 


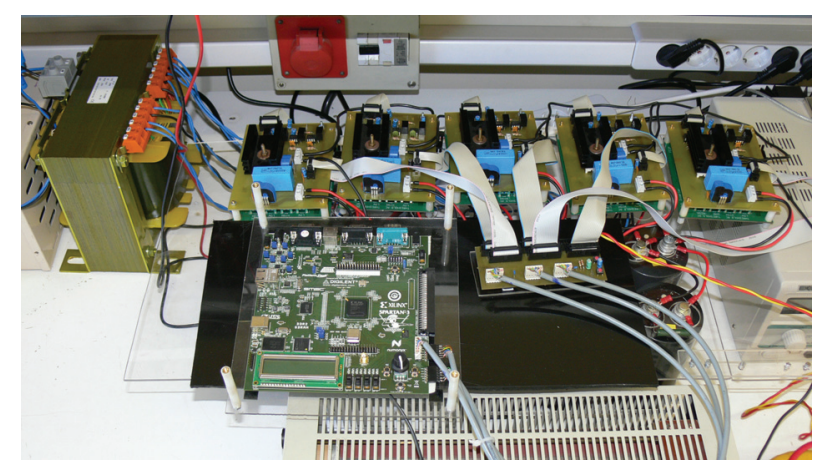

Fig. 8. Photograph of the experimental set-up.

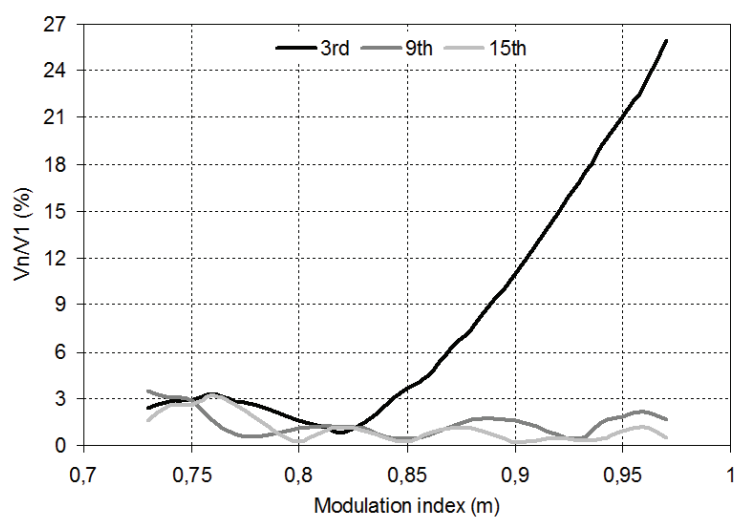

Fig. 9. Normalized amplitude of odd harmonics multiples of three for 5 H-bridges (exp.).



Fig. 10. Normalized amplitude of odd harmonics non-multiples of three for $5 \mathrm{H}$-bridges (exp.).

THD, plotted in Fig. 11, has a minimum absolute value for low modulation indexes and, as already verified in the simulations, rapidly increases to higher values for high modulation indexes.

Generally, the experimental results obtained are very similar to the simulations. The small differences can be associated with the DC ripple voltage, acquisition errors and calculation method of the THD.

\section{A. Discussion}

The method was also simulated with 3 and 7 H-bridges and experimentally tested with 3 bridges.

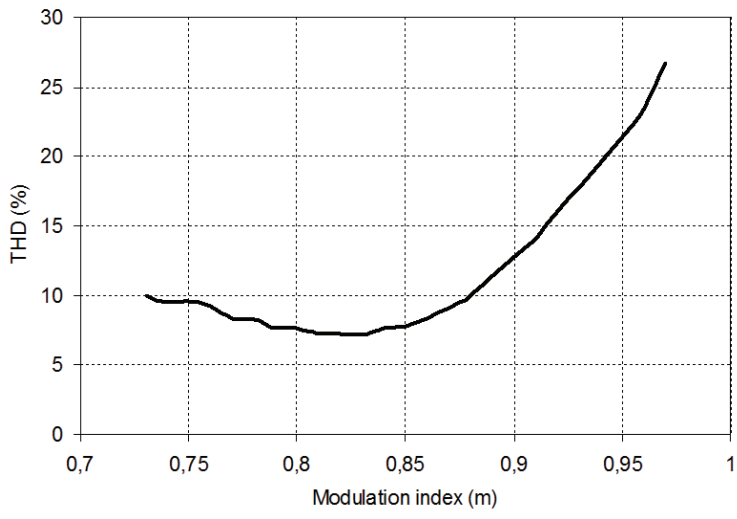

Fig. 11. THD of the output voltage for $5 \mathrm{H}$-bridges (exp.).

In terms of fundamental component variation, its range is reduced with an increasing number of bridges. Also, the range of modulation indexes where a relatively small THD occurs is reduced for an increasing number of bridges.

Independently of the number of bridges, high modulation indexes imply high values for THD; all these conclusions are clearly demonstrated in Fig. 12 and 13. This is in contrast with the high number of degrees of freedom (switching angles) available in the control method. Although not minimizing THD, other real-time methods like the one in [11] can be used for a large number of levels; the modulation index range is higher and the loworder harmonics are smaller.

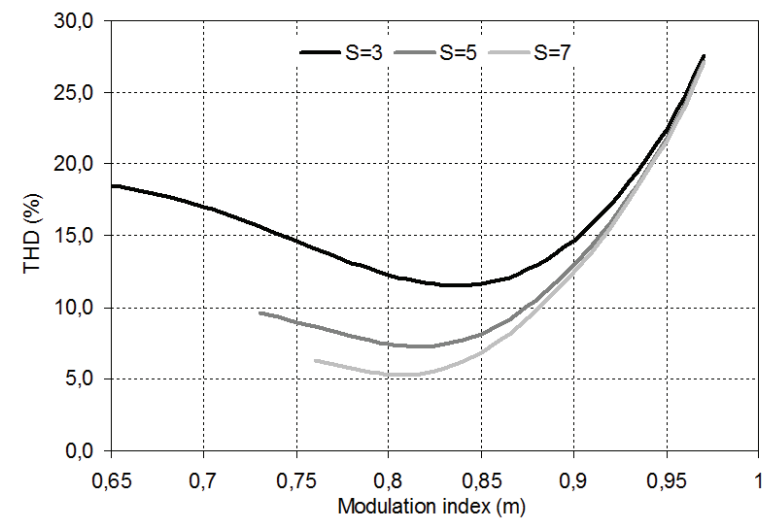

Fig. 12. THD of the output voltage for 3,5 and 7 bridges (simulation).

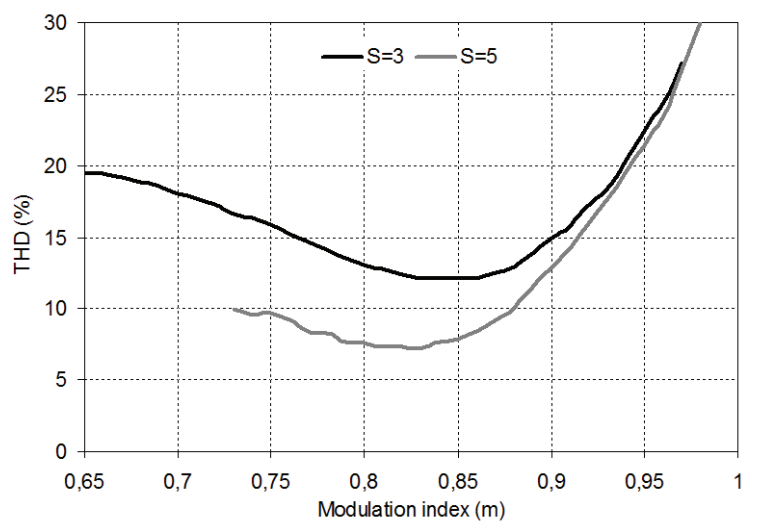

Fig. 13. THD of the output voltage for 3 and 5 bridges (experimental). 
The analyzed method allows sufficiently fast changes in amplitude and phase when compared with the expected and imposed output of a voltage control loop, and inside a relatively small range which allows the control of the converter with variations in the proximity of a given reference for the output voltage. So, applications like AC drives are not the best ones for the method; on the contrary, grid-connected converters will be well controlled.

\section{Conclusion}

The reduced number of iterations needed to obtain a solution for the switching angles allows concluding that the described method is a real-time one and can be easily implemented in a microprocessor/DSP/FPGA. From the verified characteristics of the analyzed real-time method it can be concluded that they are compatible with the interface of power electronics converters with the electric grid.

The method is still valid for a higher number of $\mathrm{H}$-bridges $(7,9, \ldots)$ but with deteriorated features, e.g. the reduction of the operating range, bringing the question of what is the limit for the number of levels where a sufficiently large amplitude range does not exist for the control of a gridconnected converter.

\section{References}

[1] B. Wu, "High Power Converters and AC Drives". Wiley/IEEE Press, New York, 2006.

[2] G. Holmes and T. Lipo, "Pulse Width Modulation for Power Converters". Wiley/IEEE Press, New York, 2003.

[3] J. Rodriguez, L. G. Franquelo, S. Kouro, J. L. Leon, R. C. Portillo, M. A. M. Prats, and M. A. Perez, "Multilevel converters: an enabling technology for high-power applications," Proceedings of the IEEE, vol. 97, no. 11, pp. 1786-1817, Nov. 2009.

[4] L. G. Franquelo, J. Rodríguez, J. I. Leon, S. Kouro, R. Portillo, and M. A. M. Prats, "The age of multilevel converters arrives", IEEE Industrial Electronics Magazine, pp. 28-39, Jun. 2008.

[5] L. M. Tolbert, F. Z. Peng, T. G. Habetler, "Multilevel converters for large electric drives", IEEE Transactions on Industry Applications, vol. 35, no.1, pp. 36-44, Jan./Feb. 1999.
[6] M. Malinowski, K. Gopakumar, J. Rodriguez, and M. A. Pérez, "A survey on cascaded multilevel inverters", IEEE Transactions on Industrial Electronics, vol. 57, no. 7, pp. 2197-2206, Jul. 2010.

[7] E. Villanueva, P. Correa, J. Rodríguez, and M. Pacas, "Control of a single-phase cascaded H-bridge multilevel inverter for grid-connected photovoltaic systems", IEEE Transactions on Industrial Electronics, vol. 56, no. 11, pp. 4399-4406, Nov. 2009.

[8] M. S. A. Dahidah, and V. G. Agelidis, "Selective harmonic elimination PWM control for cascaded multilevel voltage source converters: A generalized formula", IEEE Transactions on Power Electronics, vol. 23, no. 4, pp. 1620-1670, July 2008.

[9] J. Kumar, B. Das, and P. Agarwal, "Optimized switching scheme of a cascade multilevel inverter", Electric Power Components and Systems, vol. 38, no. 4, pp. 445-464, 2010.

[10] J. N. Chiasson, L. M. Tolbert, K. J. McKenzie, and Z. Du, "A unified approach to solving the harmonic elimination equations in multilevel converters", IEEE Transactions on Power Electronics, vol. 19, no. 2, pp. 478-490, March 2004.

[11] D.-W. Kang, H.-C. Kim, T.-J. Kim and D.-S. Hyun, "A simple method for acquiring the conducting angle in a multilevel cascaded inverter using step pulse waves", IEE Proc.-Electr. Power Appl., vol. 152, no. 1, pp. 103-111, Jan. 2005.

[12] Y. Liu, H. Hong, and A. Q. Huang, "Real-time calculation of switching angles minimizing THD for multilevel inverters with step modulation", IEEE Transactions on Industrial Electronics, vol. 56, no. 2, pp. 285-293, Feb. 2009.

[13] Y. Liu, H. Hong, and A. Q. Huang, "Real-time algorithm for minimizing THD in multilevel inverters with unequal or varying voltage steps under staircase modulation", IEEE Transactions on Industrial Electronics, vol. 56, no. 6, pp. 2249-2258, June 2009.

[14] B. Ozpineci, L. M. Tolbert, and J. N. Chiasson, "Harmonic optimization of multilevel converters using genetic algorithms," IEEE Power Electronics Letters, vol. 3, no. 3 , pp. 92-95, Sep. 2005.

[15] H. Taghizadeh, M. T. Hagh, "Harmonic elimination of cascade multilevel inverters with non-equal DC sources using particle swarm optimization", IEEE Transactions on Industrial Electronics, vol. 57, no. 11, pp. 3678-3684, Nov. 2010. 